\title{
Thermal properties of spacetime foam
}

\author{
Luis J. Garay \\ Instituto de Matemáticas y Física Fundamental, CSIC, C/ Serrano 121, 28006 Madrid, Spain
}

(Received 9 June 1998; published 17 November 1998)

\begin{abstract}
Spacetime foam can be modeled in terms of nonlocal effective interactions in a classical nonfluctuating background. Then, the density matrix for the low-energy fields evolves, in the weak-coupling approximation, according to a master equation that contains a diffusion term. Furthermore, it is argued that spacetime foam behaves as a quantum thermal field that, apart from inducing loss of coherence, gives rise to effects such as gravitational Lamb and Stark shifts as well as quantum damping in the evolution of the low-energy observables. These effects can be, at least in principle, experimentally tested. [S0556-2821(98)04524-X]
\end{abstract}

PACS number(s): 04.60.-m, 03.65.Bz, 04.20.Gz, 04.70.Dy

\section{INTRODUCTION}

It seems natural to assume that spacetime at the Planck scale must have a very complicated and ever-changing topology. Indeed, it was Wheeler [1] who suggested the foamlike structure of spacetime [1-3] as an inescapable ingredient of the yet-to-be-built quantum theory of gravity. Since then, various spacetime foam components have been proposed: wormholes [4,5], virtual black holes [6], and quantum time machines $[7,8]$ among them.

The quantum theory of gravity suffers from problems [9] that have remained unsolved for many years. They originate in the fact that gravity deals with the frame in which everything takes place, i.e., with spacetime, in sharp contrast with any other interaction, for which spacetime is a passive frame. When gravity is brought onto the scene, the frame itself becomes dynamical. It suffers the quantum fluctuations of the other interactions and, even more, introduces its own fluctuations, thus becoming an active agent in the theory.

We are use to putting everything into spacetime, so that we can name and handle events. General relativity made spacetime alive and, in this sense, was a major change. But, although dynamical, the relations between different events were still sharply defined. Quantum mechanics changed this, too. In such a dynamical frame, objects became fuzzy; exact locations were substituted by probability amplitudes of finding an object in a given region of space at a given instant of time.

A quantum uncertainty in the position of a particle implies an uncertainty in its momentum and, therefore, due to the gravity-energy universal interaction, would also imply an uncertainty in the geometry, which in turn would introduce an additional uncertainty in position of the particle. The geometry would thus be subject to quantum fluctuations that would constitute the spacetime foam and that should be of the same order as the geometry itself at the Planck scale. This would give rise to a minimum length [10] beyond which the geometrical properties of spacetime would be lost, while on larger scales it would look smooth and with a welldefined metric structure.

The quantum structure of spacetime would be relevant at energies close to Planck scale and one could expect that the quantum gravitational virtual processes that constitute the spacetime foam could not be described without knowing the details of the theory of quantum gravity. However, the gravitational nature of spacetime fluctuations provides a mechanism for studying the effects of these virtual processes in low-energy physics. Indeed, virtual gravitational collapse and topology change would forbid a proper definition of time at the Planck scale. More explicitly, in the presence of horizons, closed timelike curves, topology changes, etc., any Hamiltonian vector field that represents time evolution outside the fluctuation would vanish at points inside the fluctuation. This means that it would not be possible to describe the evolution by means of a Hamiltonian unitary flow from an initial to a final state and, consequently, quantum coherence would be lost. These effects and their order of magnitude would not depend on the detailed structure of the fluctuations but rather on their existence and global properties. In general, the regions in which the asymptotically timelike Hamiltonian vector fields vanish are associated with infinite redshift surfaces and, consequently, these small spacetime regions would behave as magnifiers of Planck length scales transforming them into low-energy modes as seen from outside the fluctuations [11]. Therefore, spacetime foam and the related minimum length would affect low-energy physics, so that low-energy experiments would effectively suffer a nonvanishing uncertainty. In this situation, a loss of quantum coherence would be almost unavoidable [12]. In fact, Hawking [6] has pointed out that scalar fields may lose coherence extremely fast and that the loss of quantum coherence might also be responsible for the vanishing of the $\theta$ angle of quantum chromodynamics.

In this paper, we show that spacetime foam behaves as a quantum thermal bath with a nearly Planckian temperature that has a weak interaction with low-energy fields. As a consequence, other effects, apart from a loss of coherence, such as Lamb and Stark transition-frequency shifts, quantum damping, and cold diffusion, characteristic of systems in a quantum environment $[13,14]$, naturally appear as lowenergy predictions of this model. A brief account of these results has already appeared in Ref. [17]. This kind of quantum gravitational effects can be, in principle, experimentally tested (see, e.g., Refs. $[15,16]$ ), as we also argue in this work.

This paper is organized as follows. In Sec. II, we propose an effective model of spacetime foam in terms of nonlocal interactions and argue that it is equivalent to a local theory with a stochastic classical Gaussian noise source. In Sec. III, 
a master equation for the low-energy density matrix is obtained and the diffusion term is studied. Section IV is devoted to the quantum effects that have not been taken into account in previous sections and derive a master equation that includes them. It is also shown that spacetime foam can be described as a quantum thermal bath and the consequences of this effective behavior are analyzed. We close this section with a short discussion about the kind of experiments and observations that could be sensitive enough to test these effects. In Sec. V, we study the role that some of the components of spacetime foam (wormholes, virtual black holes and quantum time machines) play in the effective theory. We summarize and conclude in Sec. VI.

\section{EFFECTIVE INTERACTIONS}

In this section, we will construct an effective theory for the evolution of low-energy fields in spacetime foam, where we possibly have a finite resolution limit because the notion of distance is not valid at the quantum gravitational scale.

With this aim, we will substitute spacetime foam by a fixed classical nonfluctuating background with low-energy fields living on it. We will perform a $3+1$ foliation of the effective spacetime that, for simplicity, will be regarded as flat, $t$ denoting the time parameter and $x$ the spatial coordinates. Spacetime foam features, i.e., the gravitational fluctuations and the minimum length generated by them, will be characterized by nonlocal interactions. They will relate spacetime points that are sufficiently close in the effective nonfluctuating background, where a well-defined notion of distance exists. These effective nonlocal interactions will be described in terms of local interactions as follows.

Let us consider a basis $\left\{h_{i}(t)\right\}$ of local gauge-invariant interactions at the spacetime point $(x, t)$, each element consisting of factors of the form $l_{*}^{2 n(1+s)-4}[\phi(x, t)]^{2 n}$, and $\phi$ being the low-energy field strength of spin $s$. As a notational convention, each index $i$ implies a dependence on the spatial position $x$ by default; whenever the index $i$ does not carry an implicit spatial dependence, it will appear as underlined $\underline{i}$. Also, any contraction of indices (except for underlined ones) will entail an integral over spatial positions. Then, the nonlocal effective interaction can be included in the Euclidean action by means of a term of the form

$$
I_{\mathrm{int}}=\sum_{N} \mathcal{I}_{N}
$$

where $I_{\text {int }}$ is the $N$-local interaction term,

$$
\mathcal{I}_{N}=\frac{1}{N !} \int d t_{1} \cdots d t_{N} c^{i_{1} \cdots i_{N}}\left(t_{1} \ldots t_{N}\right) h_{i_{1}}\left(t_{1}\right) \cdots h_{i_{N}}\left(t_{N}\right) \text {. }
$$

The dimensionless functions $c^{i_{1} \cdots i_{N}}\left(t_{1} \ldots t_{N}\right)$ cannot depend on the location of the gravitational fluctuation itself because of conservation of energy and momentum: the fluctuations do not carry energy, momentum, or gauge charges. Thus, diffeomorphism invariance is preserved, at least at lowenergy scales, provided that the coefficients $c^{i_{1} \cdots i_{N}}\left(t_{1} \ldots t_{N}\right)$ only depend on relative positions. This invariance cannot be expected to hold at the Planck scale as well. However, this violation of energy-momentum conservation is safely kept within Planck scale limits [18], where the processes will no longer be Markovian.

The coefficients $c^{i_{1} \cdots i_{N}}\left(t_{1} \ldots t_{N}\right)$ must vanish for relative spacetime distances larger than the length scale $r$ of the gravitational fluctuations. Indeed, if the gravitational fluctuations are smooth in the sense that they only involve trivial topologies or contain no horizons, the coefficients $c^{i_{1} \cdots i_{N}}\left(t_{1} \ldots t_{N}\right)$ will be $N$-point propagators which, as such, will have infinitely long tails and the size of the gravitational fluctuations will be effectively infinite. In other words, we would be dealing with a local theory written in a nonstandard way. The gravitational origin of these fluctuations eliminate these long tails because of the presence of gravitational collapse and topology change. This means that, for instance, virtual black holes [6] will appear and disappear and horizons will be present throughout. As Padmanabhan [11] has also argued, horizons induce nonlocal interactions of finite range since the Planckian degrees of freedom will be magnified by the horizon (because of an infinite redshift factor) thus giving rise to low-energy interactions as seen from outside the gravitational fluctuation. Virtual black holes represent a kind of components of spacetime foam that, because of the horizons and their nontrivial topology, will induce nonlocal interactions but, most probably, other fluctuations with complicated topology will warp spacetime in a similar way and the same magnification process will also take place.

Finally, the coefficients $c^{i_{1} \cdots i_{N}}\left(t_{1} \ldots t_{N}\right)$ will contain a factor $\left[e^{-S(r) / 2}\right]^{N}, S(r)$ being the Euclidean action of the gravitational fluctuation, which is of the order $\left(r / l_{*}\right)^{2}$. This is just an expression of the idea that inside large fluctuations, interactions that involve a large number of spacetime points are strongly suppressed. As the size of the fluctuation decreases, the probability for events in which three or more spacetime points are correlated increases, in close analogy with the kinetic theory of gases: the higher the density of molecules in the gas, the more probable is that a large number of molecules collide at the same point. The expansion parameter in this example is typically the density of molecules. In our case, the natural expansion parameter is the transition amplitude. It is given by the square root of the two-point transition probability which in the semiclassical approximation is of the form $e^{-S(r)}$.

A simple calculation shows that

$$
\mathcal{I}_{N} \sim \epsilon^{N}(r / l)^{2 N-4} \prod_{\underline{i}=1}^{N}\left(l_{*} / l\right)^{2 n_{\underline{i}}\left(1+s_{\underline{i}}\right)-2},
$$

where $\epsilon=e^{-S(r) / 2}\left(r / l_{*}\right)^{2}$. Indeed, $\mathcal{I}_{N}$ contains a factor $\left[e^{-S(r) / 2}\right]^{N}$ coming from the coefficient $c^{i_{1} \cdots i_{N}}\left(t_{1} \ldots t_{N}\right)$, as discussed above; each interaction $h_{i}$ provides a factor $\left(l_{*} / l\right)^{2 n_{i}\left(1+s_{i}\right)} l_{*}^{-4}$; there are also $N$ integrals over spacetime positions, $N-1$ of which are integrals over relative positions and therefore give a factor $r^{4}$ each; and, finally, the integral over the global spacetime position provides an additional factor $l^{4}$. The interaction term $\mathcal{I}_{N}$ has contributions from three different length scales, Planck length $l_{*}$, the size of the 
gravitational fluctuations $r$, and the low-energy length scale $l$, through the ratios $r / l_{*}, r / l$ and $l / l_{*}$ : the factor $\epsilon^{N}(r / l)^{2 N-4}$ depends only on the first two and is common to all powers $n$ and spins $s$ while the factors $\left(l_{*} / l\right)^{2 n_{i}\left(1+s_{\underline{i}}\right)-2}$ depend only on the low-energy scale (in Planck units) and contain information about the different kind of interactions involved.

The contributions of the trilocal and higher effective interactions are, at most, of order $\epsilon^{3}$. Therefore, in the weakcoupling approximation, i.e., up to second order in the expansion parameter $\epsilon$, they can be ignored. On the other hand, the local terms $\mathcal{I}_{0}$ and $\mathcal{I}_{1}$ can be absorbed in the bare action. Indeed, the coefficient $c$ appearing in $\mathcal{I}_{0}$ is constant; the coefficients $c^{i}(t)$ in $\mathcal{I}_{1}$ cannot depend on spacetime positions because of diffeomorphism invariance and are therefore constant as well. Consequently, we can write the nonlocal interaction term in the Euclidean action as the bilocal contribution

$$
I_{\mathrm{int}}=\frac{1}{2} \int d t d t^{\prime} c^{i j}\left(t-t^{\prime}\right) h_{i}(t) h_{j}\left(t^{\prime}\right)
$$

where we have renamed $c^{i j}\left(t, t^{\prime}\right)$ as $c^{i j}\left(t-t^{\prime}\right)$. This coefficient is symmetric in the pair of indices $i j$ and depends on the spatial positions $x_{i}$ and $x_{j}$ only through the relative distance $\left|x_{i}-x_{j}\right|$. It is of order $e^{-S(r)}$ and is concentrated within a spacetime region of size $r$.

The effect of a single spacetime fluctuation can be described in the path integral approach by adding a contribution $\int \mathcal{D} \phi e^{-I_{0}} I_{\text {int }}$ to the bare low-energy Euclidean path integral $\int \mathcal{D} \phi e^{-I_{0}}, I_{0}$ being the bare low-energy action. If we consider $\mathcal{N}$ indistinguishable gravitational fluctuations, the

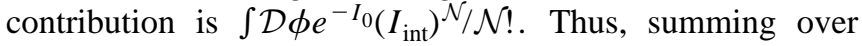
any number $\mathcal{N}$ of them, we obtain the path integral $\int \mathcal{D} \phi e^{-I_{0}+I_{\text {int }}}$.

The bilocal effective action above does not lead to a unitary evolution for the low-energy fields because there exist different trajectories that arrive at a given configuration $(\phi, \dot{\phi})$; the future evolution depends on these past trajectories and not only on the values of $\phi$ and $\dot{\phi}$ at that instant of time. Therefore, it is not sufficient to know the fields and their time derivatives at an instant of time in order to know their values at a later time: we need to know the history of the system, at least for a time $r$. As a consequence, the system cannot possess a well-defined Hamiltonian vector field and undergoes an intrinsic loss of predictability [19].

The exponential of the interaction term $e^{I_{\text {int }}}$ can be written as $[20]$

$$
\int \mathcal{D} \alpha e^{-(1 / 2) \int d t d t^{\prime} \gamma_{i j}\left(t-t^{\prime}\right) \alpha^{i}(t) \alpha^{j}\left(t^{\prime}\right)} e^{-\int d t \alpha^{i}(t) h_{i}(t)}
$$

where, the continuous matrix $\gamma_{i j}\left(t-t^{\prime}\right)$ is the inverse of $c^{i j}\left(t-t^{\prime}\right)$, i.e.,

$$
\int d t^{\prime \prime} \gamma_{i k}\left(t-t^{\prime \prime}\right) c^{k j}\left(t^{\prime \prime}-t^{\prime}\right)=\delta_{i}^{j} \delta\left(t-t^{\prime}\right)
$$

Note that the quadratic character of the distribution for the fields $\alpha^{i}$ is a consequence of the weak-coupling approximation (second order in $\epsilon$ ), which keeps only the bilocal term in the action. Beyond the weak-coupling approximation, higher-order terms would introduce deviations from this noise distribution. Note also that we have a different field $\alpha^{i}$ for each kind of interaction $h_{i}$. Thus, we have transferred the nonlocality of the low-energy fields $\phi$ to the set of fields $\alpha^{i}$, which are nontrivially coupled to it.

If we now perform a Wick rotation back to Lorentzian spacetime, we see that the path integral has the form

$$
\int \mathcal{D} \alpha P[\alpha] \int \mathcal{D} \phi e^{i\left[S_{0}+\int d t \alpha^{i}(t) h_{i}(t)\right]},
$$

where $S_{0}$ is the low-energy Lorentzian action and

$$
P[\alpha]=e^{-(1 / 2) \int d t d t^{\prime} \gamma_{i j}\left(t-t^{\prime}\right) \alpha^{i}(t) \alpha^{j}\left(t^{\prime}\right)} e^{-\int d t \alpha^{i}(t) h_{i}(t)}
$$

is the Gaussian probability distribution with correlation functions $c^{i j}\left(t-t^{\prime}\right)$ for the stochastic nonlocal fields $\alpha^{i}$ that represent spacetime foam and which are not affected by the Wick rotation.

\section{CLASSICAL DIFFUSION}

The analysis of the previous section ignores in a way the quantum nature of gravitational fluctuations such as virtual black holes or quantum time machines. Indeed, the fields $\alpha^{i}$ represent quantum gravitational spacetime foam but, as we have seen, the path integral for the whole system does not contain any trace of the dynamical character of the fields $\alpha^{i}$. It just contains a Gaussian probability distribution for them. The path integral above can then be interpreted as a Gaussian average over the classical noise sources $\alpha^{i}$. Classicality here means that we can keep the sources $\alpha^{i}$ fixed, ignoring the noise commutation relations in a kind of zeroth-order semiclassical approximation, and, at the end of the calculations, we just average over them. The next section will be devoted to the quantum noise effects generated by spacetime foam that we are ignoring here.

The master equation that governs the Lorentzian dynamics of the low-energy fields in foamlike spacetimes is derived in what follows. For each fixed set of fields $\alpha^{i}$, the evolution equation for the density matrix $\rho_{\alpha}(t)$, obtained with the Hamiltonian

$$
H_{\alpha}(t)=H_{0}+\alpha^{i}(t) h_{i}
$$

$H_{0}$ being the bare Hamiltonian of the low-energy field, is

$$
\dot{\rho}_{\alpha}(t)=-i\left[H_{0}, \rho_{\alpha}(t)\right]-i \alpha^{i}(t)\left[h_{i}, \rho_{\alpha}(t)\right] .
$$

In the interaction picture, this equation becomes

$$
\dot{\rho}_{\alpha}^{\mathrm{I}}(t)=-i \alpha^{i}(t)\left[h_{i}^{\mathrm{I}}(t), \rho_{\alpha}^{\mathrm{I}}(t)\right],
$$

where

$$
\rho_{\alpha}^{\mathrm{I}}(t)=U_{0}^{+}(t) \rho_{\alpha}(t) U_{0}(t),
$$




$$
h_{i}^{\mathrm{I}}(t)=U_{0}^{+}(t) h_{i} U_{0}(t)
$$

with $U_{0}(t)=e^{-i H_{0} t}$. Averaging this equation over $\alpha^{i}$ would provide a master equation for the density matrix of the system although it would not be very useful since it would contain terms of all orders in $\alpha^{i}$. To avoid this problem, we integrate this equation between an initial time $t_{0}$ and $t$ :

$$
\rho_{\alpha}^{\mathrm{I}}(t)=\rho_{\alpha}^{\mathrm{I}}\left(t_{0}\right)-i \int_{t_{0}}^{t} d t^{\prime} \alpha^{i}\left(t^{\prime}\right)\left[h_{i}^{\mathrm{I}}\left(t^{\prime}\right), \rho_{\alpha}^{\mathrm{I}}\left(t^{\prime}\right)\right]
$$

and introduce this formal solution back to the differential equation for $\rho_{\alpha}^{\mathrm{I}}$ noting that at the initial time $\rho_{\alpha}^{\mathrm{I}}\left(t_{0}\right)$ $=\rho^{\mathrm{I}}\left(t_{0}\right)$ does not depend on $\alpha^{i}$ (if this were not the case, there would be an extra renormalization term in the Hamiltonian):

$$
\begin{aligned}
\dot{\rho}_{\alpha}^{\mathrm{I}}(t)= & -i \alpha^{i}(t)\left[h_{i}^{\mathrm{I}}(t), \rho^{\mathrm{I}}\left(t_{0}\right)\right] \\
& -\int_{t_{0}}^{t} d t^{\prime} \alpha^{i}(t) \alpha^{j}\left(t^{\prime}\right)\left[h_{i}^{\mathrm{I}}(t),\left[h_{j}^{\mathrm{I}}\left(t^{\prime}\right), \rho_{\alpha}^{\mathrm{I}}\left(t^{\prime}\right)\right]\right] .
\end{aligned}
$$

Next, we perform the Gaussian average over $\alpha^{i}$ taking into account that $\rho_{\alpha}^{\mathrm{I}}(t)$ does not depend on $\alpha^{i}$ at zeroth order but only at first order, i.e., $\rho_{\alpha}^{\mathrm{I}}(t)=\rho^{\mathrm{I}}(t)+O(\alpha)$ with $\rho^{\mathrm{I}}(t)$ $=\left\langle\rho_{\alpha}^{\mathrm{I}}(t)\right\rangle$ and keep terms up to second order in $\epsilon$ (weakcoupling approximation). We then obtain the following equation for $\rho^{\mathrm{I}}(t)$ :

$$
\begin{aligned}
\dot{\rho}^{\mathrm{I}}(t)= & -\int_{0}^{t-t_{0}} d \tau\left\langle\alpha^{i}(t) \alpha^{j}(t-\tau)\right\rangle \\
& \times\left[h_{i}^{\mathrm{I}}(t),\left[h_{j}^{\mathrm{I}}(t-\tau), \rho^{\mathrm{I}}(t-\tau)\right]\right],
\end{aligned}
$$

where we have made a change of integration variables from $t^{\prime}$ to $\tau=t-t^{\prime}$. We also assume that $\rho^{\mathrm{I}}(t)$ hardly changes within a correlation time $r$ (Markov approximation), so that $\rho^{\mathrm{I}}(t-r) \sim \rho^{\mathrm{I}}(t)$. This amounts to ignoring terms of order $\epsilon^{4}$ in the master equation. The initial condition can be taken at $t_{0}=-\infty$, so that the integration range is now $(0, \infty)$. Note that, since $\left\langle\alpha^{i}(t) \alpha^{j}(t-\tau)\right\rangle=c^{i j}(\tau)$ is nonvanishing only for $\tau<r$, this limit $t_{0} \rightarrow-\infty$ just implies that the evolution must take place over periods of time much larger than the correlation time $r$ for the approximation to be valid. The resulting master equation in the interaction picture is then

$$
\dot{\rho}^{\mathrm{I}}(t)=-\int_{0}^{\infty} d \tau c^{i j}(\tau)\left[h_{i}^{\mathrm{I}}(t),\left[h_{j}^{\mathrm{I}}(t-\tau), \rho^{\mathrm{I}}(t)\right]\right],
$$

Transforming this equation back to the Schrödinger picture, we obtain the equation

$$
\dot{\rho}=-i\left[H_{0}, \rho\right]-\int_{0}^{\infty} d \tau c^{i j}(\tau)\left[h_{i},\left[h_{j}^{\mathrm{I}}(-\tau), \rho\right]\right] .
$$

Since $\quad h_{j}^{\mathrm{I}}(-\tau)=U_{0}^{+}(-\tau) h_{j} U_{0}(-\tau) \quad$ and $\quad U_{0}(\tau)=1$ $+O(\tau / l)$, the final form of the master equation for a low- energy system subject to gravitational fluctuations treated as a classical environment and at zeroth order in $r / l$ (the effect of higher order terms in $r / l$ will be thoroughly studied in the next section) is [21]

$$
\dot{\rho}=-i\left[H_{0}, \rho\right]-\int_{0}^{\infty} d \tau c^{i j}(\tau)\left[h_{i},\left[h_{j}, \rho\right]\right] .
$$

The first term would also be present in the absence of fluctuations, since it governs the low-energy Hamiltonian evolution. The second term is a direct consequence of the foamlike structure of spacetime and the related existence of a minimum length. It is a diffusion term which will be responsible for the loss of coherence. Note that a dissipation term, necessary to preserve the commutation relations under time evolution, is not present. However, we have considered the classical noise limit, i.e., the fields $\alpha^{i}$ have been considered as classical sources and the commutation relations are automatically preserved. We will see that the dissipation term, apart from being of quantum origin, is $r / l$ times smaller than the diffusion term and we have only considered the zeroth order approximation in $r / l$.

The diffusion term induces a characteristic decoherence time $\tau_{d}$ that can be easily calculated. Indeed, the interaction Hamiltonian density $h_{i}$ is of order $l_{*}^{-4}\left(l_{*} / l\right)^{2 n_{i}\left(1+s_{i}\right)}$ and $c^{i j}(\tau)$ is of order $e^{-S(r)}$. Furthermore, the diffusion term contains one integral over time and two integrals over spatial positions. The integral over time and the one over relative spatial positions provide a factor $r^{4}$, since $c^{i j}(\tau)$ is different from zero only in a spacetime region of size $r^{4}$, and the remaining integral over global spatial positions provides a factor $l^{3}$, the typical low-energy spatial volume. Putting everything together, we see that the diffusion term is of order $l^{-1} \epsilon^{2} \Sigma_{\underline{i j}}\left(l_{*} / l\right)^{\eta_{\underline{i}}+\eta_{j}}$, with $\eta_{\underline{i}}=2 n_{\underline{\underline{i}}}\left(1+s_{\underline{i}}\right)-2$. This quantity defines the inverse of the decoherence time $\tau_{d}$. Therefore, the ratio between the decoherence time $\tau_{d}$ and the lowenergy length scale $l$ is

$$
\tau_{d} / l \sim \epsilon^{-2}\left[\sum_{\underline{i} j}\left(l_{*} / l\right)_{\underline{\underline{j}}}+\eta_{\underline{j}}\right]^{-1}
$$

Only gravitational fluctuations whose size is very close to Planck length will give a sufficiently small decoherence time, because of the exponential dependence of $\epsilon$ $\sim e^{-S(r) / 2}\left(r / l_{*}\right)^{2}$. Slightly larger fluctuations will have a very small effect on the unitarity of the effective theory. For the interaction term that corresponds to the mass of a scalar field, the parameter $\eta$ vanishes and, consequently, $\tau_{d} / l$ $\sim \epsilon^{-2}$. Thus, the scalar mass term will lose coherence faster than any other interaction. Indeed, for higher spins and/or powers of the field strength, $\eta \geqslant 1$ and therefore $\tau_{d} / l$ increases by powers of $l / l_{*}$. For instance, the scalar-fermion interaction term $\phi^{2} \bar{\psi} \psi$, which has the next relevant decoherence time, corresponds to a decoherence ratio $\tau_{d} / l$ $\sim \epsilon^{-2} l / l_{*}$. We see that the decoherence time for the mass of scalars is independent of the low-energy length scale and, for gravitational fluctuations of size close to Planck length, $\epsilon$ may be not too small so that scalar masses may lose coher- 
ence fairly fast, maybe in a few times the typical evolution scale. Higher power and/or spin interactions will lose coherence much slower but for sufficiently high energies $l^{-1}$, although much smaller than the gravitational fluctuations energy $r^{-1}$, the decoherence time may be small enough. This means that quantum fields will lose coherence faster for higher-energy regimes.

\section{QUANTUM BATH}

In this section, we will take into account the quantum dynamical character of the fields $\alpha^{i}$ that represent spacetime gravitational fluctuations (e.g., virtual black holes or quantum time machines) and describe spacetime foam in terms of a quantum thermal bath. By comparing the system consisting of low-energy fields suitably coupled to a quantum bath $[13,14]$ with the results obtained above for gravitational fluctuations, we will see that spacetime foam can be substituted by an effective quantum thermal bath.

Let us start studying a system with a Hamiltonian

$$
H=H_{0}+H_{\text {int }}+H_{\mathrm{b}},
$$

where $H_{0}$ is the bare Hamiltonian that represents the lowenergy fields and $H_{\mathrm{b}}$ is the Hamiltonian of a bath that, for simplicity, will be represented by a real massless scalar field. The interaction Hamiltonian will be chosen to have the form $H_{\text {int }}=\xi^{i} h_{i}$, the noise operators $\xi^{i}$ being given by

$$
\xi^{i}(x, t)=i \int \frac{d k}{\sqrt{\omega}} \chi^{i}(\omega)\left[a^{+}(k) e^{i(\omega t-k x)}-a(k) e^{-i(\omega t-k x)}\right] .
$$

In this expression, $a$ and $a^{+}$are, respectively, the annihilation and creation operators associated with the bath, $\omega$ $=\sqrt{k^{2}}$, and $\chi^{i}(\omega)$ are real functions that represent the coupling between the system and the bath for each frequency $\omega$ and for each interaction $h_{i}$. These couplings $\chi^{i}(\omega)$ can also be written in the position representation if we note that the momentum of the bath scalar field $p(x, t)$ has the form

$$
p(x, t)=i \int d k \sqrt{\omega}\left[a^{+}(k) e^{i(\omega t-k x)}-a(k) e^{-i(\omega t-k x)}\right],
$$

so that the noise operators $\xi^{i}$ have the form

$$
\xi^{i}(x, t)=\int d x^{\prime} \chi^{i}\left(x-x^{\prime}\right) p\left(x^{\prime}, t\right) .
$$

Here,

$$
\chi^{\underline{i}}(y)=\int \frac{d k}{\sqrt{\omega}} \chi^{\underline{i}}(\omega) \cos (k y)
$$

represents the couplings between the low-energy fields and the bath in the position representation. Since we are trying to construct a model for spacetime foam, we will assume that the couplings $\chi^{i}(y)$ will be concentrated on a region of ra- dius $r$ and therefore the couplings $\chi^{i}(\omega)$ will induce a significant interaction with all the bath frequencies $\omega$ up to the natural cutoff $r^{-1}$. Furthermore, these couplings have dimensions of length and we will also assume that they are of order $e^{-S(r) / 2} r$. All the relevant information about the couplings is encoded in the commutation relations and the correlation function of the noise operators $\xi^{i}$.

Let us start with the commutation relations at different times of the noise variables. Taking into account the commutation relations for the annihilation and creation operators $a$ and $a^{+}$, i.e.,

$$
\begin{aligned}
{\left[a(k), a\left(k^{\prime}\right)\right] } & =\left[a^{+}(k), a^{+}\left(k^{\prime}\right)\right]=0, \\
{\left[a(k), a^{+}\left(k^{\prime}\right)\right] } & =\delta\left(k-k^{\prime}\right),
\end{aligned}
$$

it is easy to see that

$$
\left[\xi^{i}(t), \xi^{j}\left(t^{\prime}\right)\right]=i f^{i j}\left(t-t^{\prime}\right),
$$

where

$$
\begin{aligned}
f^{i j}(\tau) & =\int_{0}^{\infty} d \omega G^{i j}(\omega) \cos (\omega \tau), \\
G^{i j}(\omega) & =8 \pi \frac{\sin \left(\omega\left|x_{\underline{i}}-x_{j}\right|\right)}{\omega\left|x_{\underline{i}}-x_{\underline{j}}\right|} \chi^{i}(\omega) \chi^{j}(\omega) .
\end{aligned}
$$

Note that the functions $G^{i j}(\omega)$ and, hence, $f^{i j}(\tau)$ depend on the relative spatial distance $\left|x_{i}-x_{j}\right|$, are symmetric in the pair of indices $i j$ and are uniquely determined by the couplings $\chi^{i}(\omega)$ and vice versa. In particular, they are completely independent of the state of the bath or the system.

In order to compare this model with that of topological fluctuations previously described, it is convenient to introduce the so-called commutative noise representation [13] by defining new noise operators $\alpha^{i}$ in the following form:

$$
\alpha^{i}(t) Q\left(t^{\prime}\right) \equiv \frac{1}{2}\left[\xi^{i}(t), Q\left(t^{\prime}\right)\right]_{+}
$$

for any operator $Q$. As we have seen, the commutators of the noise operators $\xi^{i}$ at different times are $c$ numbers. Therefore, it is straightforward to check that the operators $\alpha^{i}$ commute at any time, i.e.,

$$
\left[\alpha^{i}(t), \alpha^{j}\left(t^{\prime}\right)\right]=0 .
$$

However, the commutator of $\alpha^{i}$ with any low-energy operator $A$ is in general nonvanishing and has the form:

$$
\left[A(t), \alpha^{i}\left(t^{\prime}\right)\right]=\int_{0}^{t} d \tau\left[A(t), h_{j}(\tau)\right] \dot{f}^{i j}\left(t^{\prime}-\tau\right)
$$

with $h_{i}(t)=\mathcal{U}^{+}(t) h_{i} \mathcal{U}(t)$ and $\mathcal{U}(t)=e^{-i H t}$. The function $f^{i j}(\tau)$ can be interpreted as a kind of memory function. Indeed, these commutators are nonzero for low-energy operators that are in the future or, at most, in the near past of the noise and vanish only when they are in the far past. Only in the so-called first Markov approximation the frontier among 
both regimes is sharply located where both noise and lowenergy fields are at the same instant of time.

We are now ready, following similar steps to those outlined in the previous section, to write down the master equation for the low-energy density matrix. We will describe the whole system (low-energy field and bath) by a density matrix $\rho_{T}(t)$. We will assume that, initially, the low energy fields and the bath are independent, i.e., that at the time $t_{0}$

$$
\rho_{\mathrm{T}}\left(t_{0}\right)=\rho\left(t_{0}\right) \otimes \rho_{\mathrm{b}} .
$$

As in the classical noise case, if the low-energy fields and the bath do not decouple at any time, an extra renormalization term should be added to the Hamiltonian. In the interaction picture, the density matrix has the form

$$
\rho_{\mathrm{T}}^{\mathrm{I}}(t)=U^{+}(t) \rho_{\mathrm{T}}(t) U(t),
$$

with $U(t)=U_{0}(t) U_{\mathrm{b}}(t)$, where $U_{0}(t)=e^{-i H_{0} t}$ and $U_{\mathrm{b}}(t)$ $=e^{-i H_{\mathrm{b}} t}$. It obeys the equation of motion

$$
\dot{\rho}_{\mathrm{T}}^{\mathrm{I}}(t)=-i\left[\xi^{i}(t) h_{i}^{\mathrm{I}}(t), \rho_{\mathrm{T}}^{\mathrm{I}}(t)\right]
$$

Here,

$$
\begin{aligned}
& \xi^{i}(t)=U^{+}(t) \xi^{i} U(t)=U_{\mathrm{b}}^{+}(t) \xi^{i} U_{\mathrm{b}}(t), \\
& h_{i}^{\mathrm{I}}(t)=U^{+}(t) h_{i} U(t)=U_{0}^{+}(t) h_{i} U_{0}(t) .
\end{aligned}
$$

Integrating this evolution equation and introducing the result back into it, we obtain the following integro-differential equation:

$$
\begin{aligned}
\dot{\rho}_{\mathrm{T}}^{\mathrm{I}}(t)= & -i\left[\xi^{i}(t) h_{i}^{\mathrm{I}}(t), \rho_{\mathrm{T}}^{\mathrm{I}}\left(t_{0}\right)\right] \\
& -\int_{t_{0}}^{t} d t^{\prime}\left[\xi^{i}(t) h_{i}^{\mathrm{I}}(t),\left[\xi^{j}\left(t^{\prime}\right) h_{j}^{\mathrm{I}}\left(t^{\prime}\right), \rho_{\mathrm{T}}^{\mathrm{I}}\left(t^{\prime}\right)\right]\right] .
\end{aligned}
$$

If we now trace over the variables of the bath, define $\rho^{\mathrm{I}}(t)$ $\equiv \operatorname{tr}_{\mathrm{b}}\left[\rho_{\mathrm{T}}^{\mathrm{I}}(t)\right]$ and note that $\operatorname{tr}_{\mathrm{b}}\left[\xi^{i}(t) h_{i}^{\mathrm{I}}(t) \rho_{\mathrm{T}}^{\mathrm{I}}\left(t_{0}\right)\right]=0$ (because $\left.\operatorname{tr}_{\mathrm{b}}\left[\xi^{i}(t) \rho_{\mathrm{b}}\right]=0\right)$, we obtain

$$
\dot{\rho}^{\mathrm{I}}(t)=-\int_{t_{0}}^{t} d t^{\prime} \operatorname{tr}_{\mathrm{b}}\left\{\left[\xi^{i}(t) h_{i}^{\mathrm{I}}(t),\left[\xi^{j}\left(t^{\prime}\right) h_{j}^{\mathrm{I}}\left(t^{\prime}\right), \rho_{\mathrm{T}}^{\mathrm{I}}\left(t^{\prime}\right)\right]\right]\right\} .
$$

In the weak-coupling approximation, which implies that $\xi^{i} h_{i}$ is much smaller than $H_{0}$ and $H_{\mathrm{b}}$ (this is justified since it is of order $\epsilon$ ), we assume that the bath density matrix does not change because of the interaction, so that $\rho_{\mathrm{T}}^{\mathrm{I}}(t)=\rho^{\mathrm{I}}(t)$ $\otimes \rho_{\mathrm{b}}$. The error introduced by this substitution is of order $\epsilon$ and ignoring it in the master equation amounts to keeping terms only up to second order in this parameter. Since $\left[\xi^{i}(t), h_{j}^{\mathrm{I}}\left(t^{\prime}\right)\right]=0$ because $\left[\xi^{i}, h_{j}\right]=0$, the right-hand side of this equation can be written in the following way:

$$
\begin{aligned}
& -\frac{1}{2} \int_{t_{0}}^{t} d t^{\prime}\left\{\left\langle\left[\xi^{i}(t), \xi^{j}\left(t^{\prime}\right)\right]_{+}\right\rangle\left[h_{i}^{\mathrm{I}}(t),\left[h_{j}^{\mathrm{I}}\left(t^{\prime}\right), \rho^{\mathrm{I}}\left(t^{\prime}\right)\right]\right]\right. \\
& \left.+\left\langle\left[\xi^{i}(t), \xi^{j}\left(t^{\prime}\right)\right]\right\rangle\left[h_{i}^{\mathrm{I}}(t),\left[h_{j}^{\mathrm{I}}\left(t^{\prime}\right), \rho^{\mathrm{I}}\left(t^{\prime}\right)\right]_{+}\right]\right\},
\end{aligned}
$$

where the average of any operator $Q$ has been defined as $\langle Q\rangle \equiv \operatorname{tr}_{\mathrm{b}}\left(Q \rho_{\mathrm{b}}\right)$. Next we note that $\left\langle\left[\xi^{i}(t), \xi^{j}\left(t^{\prime}\right)\right]\right\rangle=i f^{i j}(t$ $\left.-t^{\prime}\right)$ and, using the commutative noise representation introduced above, we can write

$$
\frac{1}{2}\left\langle\left[\xi^{i}(t), \xi^{j}\left(t^{\prime}\right)\right]_{+}\right\rangle=\left\langle\alpha^{i}(t) \alpha^{j}\left(t^{\prime}\right)\right\rangle \equiv c^{i j}\left(t-t^{\prime}\right) .
$$

If we make the assumption that the bath is in a thermal state $\rho_{\mathrm{b}}=\mathcal{Z}^{-1} e^{-H_{\mathrm{b}} / T}$ with a temperature inversely proportional to the size of the gravitational fluctuations (e.g., the radius of the virtual black holes, or the size of the regions containing closed timelike curves in the case of quantum time machines), $T \sim 1 / r$, the correlation function $c^{i j}\left(t-t^{\prime}\right)$ acquires the form:

$$
c^{i j}(\tau)=\int_{0}^{\infty} d \omega \omega G^{i j}(\omega)[N(\omega)+1 / 2] \cos (\omega \tau),
$$

where $N(\omega)=[\exp (\omega / T)-1]^{-1}$ is the mean occupation number of the bath corresponding to the frequency $\omega$. In this calculation, we have made use of the following relations, valid for a thermal state:

$$
\begin{aligned}
\langle a(k)\rangle & =\left\langle a^{+}(k)\right\rangle=0, \\
\left\langle a(k) a\left(k^{\prime}\right)\right\rangle & =\left\langle a^{+}(k) a^{+}\left(k^{\prime}\right)\right\rangle=0, \\
\left\langle a^{+}(k) a\left(k^{\prime}\right)\right\rangle & =N(\omega) \delta\left(k-k^{\prime}\right) .
\end{aligned}
$$

Similarly, we can easily compute the higher order correlations $\left\langle\alpha^{i}(t) \alpha^{j}\left(t^{\prime}\right) \alpha^{k}\left(t^{\prime \prime}\right)\right\rangle$, etc. Those containing an odd number of fields $\alpha^{i}$ turn out to be identically zero while those containing an even number can be written in terms of the two-point correlation function $c^{i j}(\tau)$. This means that the trace $\langle Q\rangle$ corresponds to a Gaussian average over $\alpha^{i}$, provided that the bath is in a thermal state, as we are considering. In this way, we have established a relation between a quantum thermal bath and spacetime foam, which can also be described by a Gaussian average, as we have seen.

The Markov approximation allows the substitution of $\rho^{\mathrm{I}}\left(t^{\prime}\right)$ by $\rho^{\mathrm{I}}(t)$ in the master equation because the integral over $t^{\prime}$ will get a significant contribution from times $t^{\prime}$ that are close to $t$ due to the factors $\dot{f}^{i j}\left(t-t^{\prime}\right)$ and $c^{i j}\left(t-t^{\prime}\right)$ and because, in this interval of time, the density matrix $\rho^{\mathrm{I}}$ will not change significantly. Indeed, the typical evolution time of $\rho^{\mathrm{I}}$ is the low-energy time scale $l$, which will be much larger than the time scale $r$ associated with the bath. If we perform a change of the integration variable from $t^{\prime}$ to $\tau$ $=t-t^{\prime}$, write

$$
\rho^{\mathrm{I}}\left(t^{\prime}\right)=\rho^{\mathrm{I}}(t-\tau)=\rho^{\mathrm{I}}(t)-\tau \dot{\rho}^{\mathrm{I}}(t)+O\left(\tau^{2}\right),
$$


and introduce this expression in the master equation above, we easily see that the error introduced by the Markovian approximation is of order $\epsilon^{2}$, i.e., it amounts to ignoring a term of order $\epsilon^{4}$. The upper integration limit $t$ in both integrals can be substituted by $\infty$ for evolution times $t-t_{0}$ much larger than the correlation time $r$, because of the factors $\dot{f}^{i j}(\tau)$ and $c^{i j}(\tau)$ that vanish for $\tau>r$, which is equivalent to taking the initial condition to the infinite past $t_{0} \rightarrow-\infty$.

Then, the master equation in the interaction picture acquires the form

$$
\begin{aligned}
\dot{\rho}^{\mathrm{I}}(t)= & -\frac{i}{2} \int_{0}^{\infty} d \tau \dot{f}^{i j}(\tau)\left[h_{i}^{\mathrm{I}}(t),\left[h_{j}^{\mathrm{I}}(t-\tau), \rho^{\mathrm{I}}(t)\right]_{+}\right] \\
& -\int_{0}^{\infty} d \tau c^{i j}(\tau)\left[h_{i}^{\mathrm{I}}(t),\left[h_{j}^{\mathrm{I}}(t-\tau), \rho^{\mathrm{I}}(t)\right]\right] .
\end{aligned}
$$

We can now transform the resulting equation back to the Schrödinger picture

$$
\begin{aligned}
\dot{\rho}= & -i\left[H_{0}, \rho\right]-\frac{i}{2} \int_{0}^{\infty} d \tau \dot{f}^{i j}(\tau)\left[h_{i},\left[h_{j}^{\mathrm{I}}(-\tau), \rho\right]_{+}\right] \\
& -\int_{0}^{\infty} d \tau c^{i j}(\tau)\left[h_{i},\left[h_{j}^{\mathrm{I}}(-\tau), \rho\right]\right] .
\end{aligned}
$$

After an integration by parts, the second term of the righthand side becomes

$$
\frac{i}{2} f^{i j}(0)\left[h_{i} h_{j}, \rho\right]-\frac{i}{2} \int_{0}^{\infty} d \tau f^{i j}(\tau)\left[h_{i},\left[\dot{h}_{j}^{\mathrm{I}}(-\tau), \rho\right]_{+}\right] \text {. }
$$

The first term is just a finite renormalization of the original low-energy Hamiltonian from $H_{0}$ to

$$
H_{0}^{\prime}=H_{0}-\frac{1}{2} f^{i j}(0) h_{i} h_{j}
$$

and the master equation can then be written in its final form

$$
\begin{aligned}
\dot{\rho}= & -i\left[H_{0}^{\prime}, \rho\right]-\frac{i}{2} \int_{0}^{\infty} d \tau f^{i j}(\tau)\left[h_{i},\left[\dot{h}_{j}^{\mathrm{I}}(-\tau), \rho\right]_{+}\right] \\
& -\int_{0}^{\infty} d \tau c^{i j}(\tau)\left[h_{i},\left[h_{j}^{\mathrm{I}}(-\tau), \rho\right]\right] .
\end{aligned}
$$

Before discussing this equation in full detail, let us first study the classical noise limit. With this aim, let us introduce the parameter

$$
\sigma=\int d k^{\prime}\left[a(k), a^{+}\left(k^{\prime}\right)\right]
$$

which is equal to 1 for quantum noise and 0 for classical noise. Then, the $f$ term is proportional to $\sigma$ and therefore vanishes in the classical noise limit. The $c$ term also contains a factor $\sigma$ but, in addition, $N(\omega)$ becomes $N(\sigma \omega)$ when introducing the parameter $\sigma$. In the limit $\sigma \rightarrow 0$, the term proportional to $1 / 2$ in $c^{i j}(\tau)$ vanishes and the term proportional to $N(\sigma \omega)$ acquires the value $c_{\text {class }}^{i j}(\tau)=T f^{i j}(\tau)$. Also, the renormalization term of the low-energy Hamiltonian vanishes in this limit. In this way, we have arrived at the same master equation that we obtained in the previous section. This is not surprising because the origin of the $f$ term is precisely the noncommutativity of the noise operators, i.e., its quantum nature, while the $c_{\text {class }}$ term actually contains the temperature effects. At zeroth order in $r / l$, the master equation for classical noise then acquires the form

$$
\dot{\rho}=-i\left[H_{0}, \rho\right]-\int_{0}^{\infty} d \tau c_{\text {class }}^{i j}(\tau)\left[h_{i},\left[h_{j}, \rho\right]\right] .
$$

Let us now analyze the general master equation, valid up to second order in $\epsilon$ that takes into account the quantum nature of the gravitational fluctuations. These contributions, although small in the low-energy regime, might still be experimentally testable. In addition, they may provide interesting information about the higher-energy regimes in which $l$ may be of the order of a few Planck lengths and for which the weak-coupling approximation is still valid. In order to see these contributions explicitly, let us further elaborate the master equation. In terms of the operator $L_{0}$ defined as $L_{0} A=\left[H_{0}, A\right]$ acting on any low-energy operator $A$, the time dependent interaction $h_{j}^{\mathrm{I}}(-\tau)$ can be written as

$$
h_{j}^{\mathrm{I}}(-\tau)=e^{-i L_{0} \tau} h_{j} .
$$

The interaction $h_{j}$ can be expanded in eigenoperators $h_{j \Omega}^{ \pm}$of the operator $L_{0}$, i.e.,

$$
h_{j}=\int d \mu_{\Omega}\left(h_{j \Omega}^{+}+h_{j \Omega}^{-}\right),
$$

with $L_{0} h_{j \Omega}^{ \pm}= \pm \Omega h_{j \Omega}^{ \pm}$and $d \mu_{\Omega}$ being an appropriate spectral measure, which is naturally cut off around the low-energy scale $l^{-1}$. This expansion always exists provided that the eigenstates of $H_{0}$ form a complete set. Then, $h_{j}^{\mathrm{I}}(-\tau)$ can be written as

$$
h_{j}^{\mathrm{I}}(-\tau)=\int d \mu_{\Omega}\left(e^{-i \Omega \tau} h_{j \Omega}^{+}+e^{i \Omega \tau} h_{j \Omega}^{-}\right) .
$$

It is also convenient to define the new interaction operators for each low-energy frequency $\Omega$ :

$$
\begin{aligned}
& h_{j \Omega}^{1}=h_{j \Omega}^{+}-h_{j \Omega}^{-}, \\
& h_{j \Omega}^{2}=h_{j \Omega}^{+}+h_{j \Omega}^{-} .
\end{aligned}
$$

Both the term proportional to $f^{i j}(\tau)$ and the term proportional to $c^{i j}(\tau)$ are integrated over $\tau \in(0, \infty)$. Because of these incomplete integrals, each term provides two different kinds of contributions coming from the bulk term and the principal part in the well-known formula

$$
\int_{0}^{\infty} d \tau e^{i \omega \tau}=\pi \delta(\omega)+\mathcal{P}(i / \omega)
$$


where $\mathcal{P}$ is the Cauchy principal part [22]. form:

The master equation can then be written in the following

$$
\dot{\rho}=-\left(i L_{0}^{\prime}+L_{\mathrm{diss}}+L_{\mathrm{diff}}+i L_{\mathrm{stark}}+i L_{\mathrm{lamb}}\right) \rho,
$$

where the meaning of the different terms is explained in what follows.

The first term $-i L_{0}^{\prime} \rho$, with $L_{0}^{\prime} \rho=\left[H_{0}^{\prime}, \rho\right]$, is responsible for the renormalized low-energy Hamiltonian evolution. The renormalization term is of order $\varepsilon^{2}$ as compared with the low-energy Hamiltonian $H_{0}$, where $\varepsilon^{2}=\epsilon^{2} \Sigma_{\underline{i j}}\left(l_{*} / l\right)^{\eta_{i}+\eta_{j}}$ and, remember, $\eta_{\underline{i}}=2 n_{\underline{i}}\left(1+s_{\underline{i}}\right)-2$ is a parameter specific to each kind of interaction term $\bar{h}_{i}$.

The dissipation term

$$
L_{\mathrm{diss}} \rho=-\frac{\pi}{4} \int d \mu_{\Omega} \Omega G^{i j}(\Omega)\left[h_{i},\left[h_{j \Omega}^{1}, \rho\right]_{+}\right]
$$

is necessary for the preservation in time of the low-energy commutators in the presence of quantum noise. As we have seen, it is proportional to the commutator between the noise creation and annihilation operators associated with the effective bath that represents spacetime foam and, therefore, vanishes in the classical noise limit. Its size is of order $\varepsilon^{2} r / l^{2}$.

The diffusion process is governed by

$$
L_{\mathrm{diff}} \rho=\frac{\pi}{2} \int d \mu_{\Omega} \Omega G^{i j}(\Omega)[N(\Omega)+1 / 2]\left[h_{i},\left[h_{j \Omega}^{2}, \rho\right]\right],
$$

which contains two contributions: the first one is a temperature effect of order $\varepsilon^{2} / l$ and the second is a cold diffusion originated in the vacuum fluctuations of the gravitational field and it is of order $\varepsilon^{2} r / l^{2}$. In the classical noise limit, only the first contribution survives and was already studied in the previous section.

The next term provides an energy shift which can be interpreted as a gravitational ac Stark effect by comparison with its quantum optical analog $[13,14]$. Its expression is

$$
\begin{aligned}
L_{\text {stark }} \rho= & \int d \mu_{\Omega} \mathcal{P} \int_{0}^{\infty} d \omega \frac{\omega \Omega}{\omega^{2}-\Omega^{2}} \\
& \times G^{i j}(\omega) N(\omega)\left[h_{i},\left[h_{j \Omega}^{1}, \rho\right]\right] .
\end{aligned}
$$

Although it is also a temperature-dependent effect with the same origin as the diffusion term, it contains a Cauchy principal part. This translates into the fact that it is smaller than the diffusion term although it does not vanish in the classical noise limit. It is of order $\varepsilon^{2} r / l^{2}$.

Finally, $L_{\mathrm{lamb}} \rho$ is an energy shift generated by the vacuum fluctuations of the gravitational field (as the dissipation term and the cold diffusion term) and that can therefore be interpreted as a gravitational Lamb shift. It has the form

$$
\begin{aligned}
L_{\mathrm{lamb}} \rho= & \frac{1}{2} \int d \mu_{\Omega} \mathcal{P} \int_{0}^{\infty} d \omega \frac{\Omega}{\omega^{2}-\Omega^{2}} G^{i j}(\omega) \\
& \times\left\{\omega\left[h_{i},\left[h_{j \Omega}^{1}, \rho\right]\right]-\Omega\left[h_{i},\left[h_{j \Omega}^{2}, \rho\right]_{+}\right]\right\} .
\end{aligned}
$$

The second term is of order $\varepsilon^{2} r^{2} / l^{3}$, which is fairly small. However, the first term will provide a significant contribution of order $\varepsilon^{2} r / l^{2} \log (l / r)$. This logarithmic dependence on the relative scale is indeed characteristic of the Lamb shift $[13,14,23]$.

As a summary, the $c$ term gives rise to four different contributions: a thermal diffusion term, another diffusion term originated from the vacuum fluctuations of the bath, a contribution to what can be interpreted as a gravitational Lamb shift, and, finally, a shift in the scalar-field oscillation frequencies that can be interpreted as a gravitational Stark effect. The $f$ term provides a dissipation part, necessary for the preservation of commutators, and another contribution to the gravitational Lamb shift. The size of these effects generated by spacetime foam, compared with the bare evolution, are the following: the thermal diffusion term is of order $\varepsilon^{2}$, which is the only one that survived in the approximations of the previous section; the diffusion created by vacuum fluctuations, the damping term, and the Stark effect are smaller by a factor $r / l$; and the Lamb shift has two contributions: one is smaller than the diffusion term by a factor $(r / l)^{2}$ and the other is of order $(r / l) \log (l / r)$ as compared with the diffusion term. Note that the quantum effects induced by spacetime foam become relevant as the low-energy length scale $l$ decreases, as we see from the fact that these effects depend on the ratio $r / l$, while, in this situation, the diffusion process becomes slower, except for the mass of scalars, which always decoheres in a time scale which is close to the lowenergy evolution time.

These quantum gravitational effects could be measured, at least in principle, since they are just energy shifts and decoherence effects similar to those appearing in other areas of physics, where fairly well established experimental procedures and results exist, and which can indeed be applied here-such as those briefly discussed below-provided that sufficiently high accuracy can be achieved. On the other hand, scalar fields lose quantum coherence extremely fast and Hawking has argued [6] that this might be the reason for not observing the Higgs particle. He has also suggested that loss of quantum coherence might be responsible for the vanishing of the $\theta$ angle in quantum chromodynamics [6].

Neutral kaon beams have been proposed as experimental systems for measuring the loss of coherence owing to quantum gravitational fluctuations $[15,24,25]$. In these systems, the main experimental consequence of the diffusion term (together with the dissipative one necessary for reaching a stationary regime) is violation of $C P T[26,12]$ because of the nonlocal origin of the effective interactions. The estimates for this violation are very close to the values accessible by current experiments with neutral kaons and will be within the range of near-future experiments. Macroscopic neutron interferometry $[15,27]$ provides another kind of experimental sys- 
tems in which the effects of the diffusion term have measurable consequences since they may cause the disappearance of the interference fringes [15,27].

As for the gravitational Lamb and Stark effects, they are energy shifts that depend on the frequency, so that different low-energy modes will undergo different shifts. This translates into a modification of the dispersion relations, which makes the velocity of propagation frequency-dependent, as if low-energy fields propagated in a "medium." Therefore, upon arrival at the detector, low-energy modes will experience different time delays (depending on their frequency) as compared to what could be expected in the absence of quantum gravitational fluctuations. These time delays in the detected signals will be very small in general. However, it is still possible to measure them if we make the low-energy particles travel large (cosmological) distances. In fact, $\gamma$-ray bursts provide such a situation as has been recently pointed out [16], thus opening a new doorway to observations of these quantum gravitational effects. Indeed, the ratio between the time delay owing to gravitational fluctuations and the width of the intrinsic time structure of $\gamma$-ray bursts has been estimated to be of order 1 for emissions with millisecond time structure and energy around $20 \mathrm{MeV}$, provided that they travel a distance of $10^{10}$ light years [16], which are compatible with $\gamma$-ray burst observations. If this sensitivity can actually be reached, one would expect that the presence of the gravitational Lamb and Stark shifts predicted above could be observationally tested.

\section{VIRTUAL BLACK HOLES, WORMHOLES, AND TIME MACHINES}

It is well-known that it is not possible to classify all fourdimensional topologies [2] and, consequently, all the possible components of spacetime foam. Here, we will briefly discuss three different kinds of fluctuations: simply connected nontrivial topologies, multiply connected topologies with trivial second homology group (i.e., with vanishing second Betti number), and finally spacetimes with a nontrivial causal structure, i.e., with closed timelike curves, in a bounded region.

The effective description proposed in this paper and the associated master equation are particularly suited to the study of low-energy effects produced by simply connected topology fluctuations (e.g., virtual black holes). Hawking [6] has shown that compact simply connected bubbles with the topology $S^{2} \times S^{2}$ (whose second Betti number is $B_{2}=1$ ) can be interpreted as closed loops of virtual black holes if one realizes [28] that the process of creation of a pair of real charged black holes accelerating away from each other in a spacetime which is asymptotic to $\mathfrak{R}^{4}$ is provided by the Ernst solution [29]. This solution has the topology $S^{2} \times S^{2}$ minus a point (which is sent to infinity) and this topology is the topological sum of the bubble $S^{2} \times S^{2}$ plus $\mathfrak{R}^{4}$. Virtual black holes will not obey classical equations of motion but will appear as quantum fluctuations of spacetime and thus will become part of the spacetime foam. Particles could fall into these black holes and be re-emitted. The scattering amplitudes of these processes [6] could be interpreted as being produced by nonlocal effective interactions that would take place inside the fluctuations and the master equation obtained above could then be interpreted as providing the evolution of the low-energy density matrix in the presence of a bath of ubiquitous quantum topological fluctuations of the virtual-black-hole type.

Wormholes [4], i.e., multiply connected fluctuations (with vanishing second Betti number), also admit a description in terms of nonlocal interactions that, in the weak-coupling approximation, become bilocal. These quantum fluctuations connect spacetime points that may be far apart from each other, in the dilute gas approximation. Therefore, diffeomorphism invariance on each spacetime region requires the coefficients $c^{i j}$ of this bilocal interaction term to be spacetime independent. The same conclusion can also be reached if we analyze wormholes from the point of view of the universal covering manifold, which is, by definition, simply connected. A wormhole is then represented in the universal covering manifold by two boundaries, suitably identified, located at infinity. This identification can be implemented by introducing coefficients $c^{i j}$ that relate the bases of the Hilbert space of wormholes in both regions of the universal covering manifold. As coefficients in a change of basis, $c^{i j}$ cannot depend on spacetime positions and, therefore, will just be constant. This means that the fields $\alpha^{i}$ cannot be interpreted as noise sources that are Gaussian distributed at each spacetime point independently, because the correlation time for the fields $\alpha^{i}$ is infinite. Indeed, the constancy of $c^{i j}$ implies that they are infinitely coherent and the Gaussian distribution to which they are subject is therefore global, spacetime independent [5]. One could still expect some effects originated in the quantum nature of $\alpha^{i}$ such as a cold diffusion term in the master equation or even dissipation. However, because they are spacetime independent, they commute with every operator, including low-energy ones, thus giving rise to superselection sectors. Therefore, all the terms in the master equation, except the one responsible for the unitary low-energy evolution, vanish. Still, wormholes can be represented by a thermal bath as we have done with localized gravitational fluctuations. However, in order to reproduce their infinite correlation time, the couplings $\xi^{i}$ between the bath and the low-energy fields must be constant, they must commute with every other operator, and, related to these two facts, only the zero-frequency (i.e., infinite wavelength) mode of the bath can be coupled to the low-energy fields, thus leading to a unitary effective theory.

From the semiclassical point of view, most of the hitherto proposed time machines [30] are unstable because quantum vacuum fluctuations generate divergences in the stressenergy tensor, i.e., are subject to the chronology protection conjecture [31]. However, quantum time machines [8] confined to small spacetime regions, for which the chronology protection conjecture does not apply [32], are likely to occur within the realm of spacetime foam, where strong causality violations or even the absence of a causal structure are expected. These Planck-size regions with quantum time machines admit an effective representation in terms of nonlocal interactions that account for the causality violations and will lead to a loss of quantum coherence [7] that can also be 
effectively described as coming from the interaction of lowenergy fields with a thermal bath. In this case, the lowenergy density matrix will also evolve according to the master equation obtained in the previous sections.

\section{CONCLUSIONS}

In this paper, we have built an effective theory in which quantum gravitational spacetime foam has been substituted by a fixed classical background plus nonlocal interactions between the low-energy fields confined to bounded spacetime regions of nearly Planck size. In the weak-coupling approximation, these nonlocal interactions become bilocal. The low-energy evolution is not unitary because of the absence of a nonvanishing timelike Hamiltonian vector field. The nonunitarity of the bilocal interaction can be encoded in a classical noise source locally coupled to the low-energy fields and subject to a Gaussian probability distribution. Then, the evolution of low-energy fields is provided by a master equation which contains a diffusion term. This diffusion is a direct consequence of the nonlocal character of the quantum gravitational fluctuations encompassed by spacetime foam. The decoherence rate is suppressed by powers of the ratio between the gravitational fluctuation size and the low-energy length scale, except for the mass interaction term of scalar fields for which this rate is comparable with the low-energy evolution scale.

We have argued that the quantum nature of spacetime foam is not represented in this effective theory but only its thermal properties. A model in terms of a quantum thermal field, which in the classical noise limit coincides with the one described above, has been proposed as describing the quantum and thermal properties of spacetime foam. In this model, the low-energy density matrix evolves according to a master equation that, apart from inducing loss of coherence, contains additional terms that may be relevant for sufficiently high energies. These terms correspond to a dissipation process that ensure the preservation of commutators, a cold diffusion, and energy shifts that can be interpreted as gravitational Lamb and Stark effects. We have also briefly discussed some of the possible experimental implications that these quantum gravitational effects may have. A constructive model in terms of nonlocal interactions that takes into account the quantum origin of spacetime foam will be developed elsewhere [33,34] within the formalism of Feynman and Vernon [35-37].

Finally, among the possible components of spacetime foam, the role of virtual black holes and small bounded regions that contain closed timelike curves has been briefly analyzed in the context of our effective model. We have also argued that dilute wormholes do not admit a description in terms of a thermal bath coupled to the whole low-energy spectrum and that they are infinitely coherent as was already shown by Coleman [5].

\section{ACKNOWLEDGMENTS}

I am very grateful to G. A. Mena Marugán, P. F. González-Díaz, C. Barceló, J. M. Raya, I. L. Egusquiza, C. Cabrillo, and J. I. Cirac for helpful discussions. I was supported by funds provided by DGICYT and MEC (Spain) under Contract Adjunct to the Project No. PB94-0107.
[1] J. A. Wheeler, Ann. Phys. (N.Y.) 2, 604 (1957); J. A. Wheeler, Geometrodynamics (Academic, London, 1962); J. A. Wheeler, in Relativity, Groups and Topology, edited by B. S. and C. M. DeWitt (Gordon and Breach, New York, 1964).

[2] S. W. Hawking, Nucl. Phys. B144, 349 (1978).

[3] S. Carlip, Phys. Rev. Lett. 79, 4071 (1997); Class. Quantum Grav. 15, 2629 (1998).

[4] S. W. Hawking, Phys. Rev. D 37, 904 (1988).

[5] S. Coleman, Nucl. Phys. B307, 867 (1988).

[6] S. W. Hawking, Phys. Rev. D 53, 3099 (1996).

[7] S. W. Hawking, Phys. Rev. D 52, 5681 (1995).

[8] P. F. González-Díaz, Phys. Rev. D (to be published) gr-qc/9712033; in Proceedings of the International Seminar on Mathematical Cosmology, edited by U. Kasper, M. Rainer, and H. J. Schmidt (World Scientific, Singapore, in press).

[9] C. J. Isham, in Proceedings of the 14th International Conference on General Relativity and Gravitation (World Scientific, Singapore, 1997).

[10] L. J. Garay, Int. J. Mod. Phys. A 10, 145 (1995).

[11] T. Padmanabhan, hep-th/9801138; hep-th/9801015.

[12] S. W. Hawking, Commun. Math. Phys. 87, 395 (1982).

[13] C. W. Gardiner, Quantum Noise (Springer-Verlag, Berlin, 1991).
[14] H. Carmichel, An Open Systems Approach to Quantum Optics (Springer-Verlag, Berlin, 1993).

[15] J. Ellis, J. S. Hagelin, D. V. Nanopoulos, and M. Srednicki, Nucl. Phys. B241, 381 (1984).

[16] G. Amelino-Camelia, J. Ellis, N. E. Mavromatos, D. V. Nanopoulos, and S. Sarkar, Nature (London) 393, 763 (1998).

[17] L. J. Garay, Phys. Rev. Lett. 80, 2508 (1998).

[18] W. G. Unruh and R. M. Wald, Phys. Rev. D 52, 2176 (1995).

[19] D. A. Eliezer and R. P. Woodard, Nucl. Phys. B325, 389 (1989).

[20] J. Zinn-Justin, Quantum Field Theory and Critical Phenomena, 3rd ed. (Oxford University Press, Oxford, 1996).

[21] T. Banks, L. Susskind, and M. E. Peskin, Nucl. Phys. B244, 125 (1984).

[22] M. Reed and B. Simon, Methods of Modern Mathematical Physics I. Functional Analysis (Academic, New York, 1972).

[23] C. Itzykson and J. B. Zuber, Quantum Field Theory (McGrawHill, Singapore, 1985).

[24] P. Huet and M. E. Peskin, Nucl. Phys. B434, 3 (1995); P. Huet, "CPT violation from Planck scale physics," hep-ph/9607435.

[25] F. Benatti and R. Floreanini, Nucl. Phys. B511, 550 (1998).

[26] D. N. Page, Gen. Relativ. Gravit. 14, 299 (1982). 
[27] A. Zeilinger, M. A. Horne, and C. G. Shull, in Proceedings of the International Symposium on the Foundations of Quantum Mechanics, edited by S. Kamefuchi et al. (Physical Society of Japan, Tokyo, 1984).

[28] G. W. Gibbons, in Fields and Geometry 1986, edited by A. Jadczyk (World Scientific, Singapore, 1986).

[29] F. J. Ernst, J. Math. Phys. 17, 515 (1976).

[30] M. J. Morris, K. S. Thorne, and U. Yurtsever, Phys. Rev. Lett. 61, 1446 (1988); M. J. Morris and K. S. Thorne, Am. J. Phys. 56, 395 (1988)

[31] S. W. Hawking, Phys. Rev. D 46, 603 (1992).
[32] Li-Xin Li and J. R. Gott III, Phys. Rev. Lett. 80, 2980 (1998). [33] L. J. Garay, (unpublished).

[34] L. J. Garay, in Proceedings of the International Seminar on Mathematical Cosmology, edited by U. Kasper, M. Rainer, and H. J. Schmidt (World Scientific, Singapore, in press).

[35] R. P. Feynman and A. R. Hibbs, Quantum Mechanics and Path Integrals (McGraw-Hill, New York, 1965).

[36] R. P. Feynman and F. L. Vernon, Ann. Phys. (N.Y.) 24, 118 (1963).

[37] A. O. Caldeira and A. J. Leggett, Physica A 121, 587 (1983). 